\title{
Reflets
}

Revue ontaroise d'intervention sociale et communautaire

\section{L'intervention contre la violence conjugale à Sudbury : aperçu et réflexions}

\section{Francine Boudreau}

Volume 2, numéro 1, printemps 1996

Contrer la violence subie par les femmes et les enfants en milieu familial

URI : https://id.erudit.org/iderudit/026107ar

DOI : https://doi.org/10.7202/026107ar

Aller au sommaire du numéro

Éditeur(s)

Reflets : Revue ontaroise d'intervention sociale et communautaire

ISSN

1203-4576 (imprimé)

1712-8498 (numérique)

Découvrir la revue

Citer cet article

Boudreau, F. (1996). L'intervention contre la violence conjugale à Sudbury: aperçu et réflexions. Reflets, 2(1), 98-108. https://doi.org/10.7202/026107ar

Tous droits réservés (C) Reflets : Revue ontaroise d'intervention sociale et communautaire, 1996
Ce document est protégé par la loi sur le droit d'auteur. L'utilisation des services d'Érudit (y compris la reproduction) est assujettie à sa politique d'utilisation que vous pouvez consulter en ligne.

https://apropos.erudit.org/fr/usagers/politique-dutilisation/ 


\title{
L'intervention contre la violence conjugale à Sudbury: aperçu et réflexions
}

\author{
F rancine B oudreau \\ $D$ irectrice $C$ linique, Service $F$ amilial de Sudbury
}

LeService Familial de Sudbury est un organismeà but non lucratif, qui a ouvert ses portes en 1971. A gence francophone vouée aux besoins de l'unité familiale, le Service Familial offre des services bilingues, sans discrimination, à tous les résidents de la région de Sudbury. Le Service Familial offre les programmes suivants: programme de counselling général, programme d'aide aux employés, et le seul programme en français d'intervention contre la violence conjugale. Dans ce court article, c'est de ce dernier programme dont nous discuterons. Plus particulièrement, nous présenterons dans un premier temps, un aperçu du programme, de ses objectifs et buts. $D$ ansun deuxième temps, noustoucherons à l'implication communautaire de l'agence afin de montrer les liens que nous entretenons avec la communauté. Finalement, compte tenu des difficultés quotidiennes que rencontrent les intervenantes et intervenants dans leur pratique, nous les aborderons afin de montrer la réalité à laquelle fait face le programme contre la violence, et les réflexions qu'elles nous posent. Les difficultés rencontrées au Service Familial n'étant pas uniques à cet organisme, nous nous interrogerons en conclusion sur les stratégies à mettre en oeuvre en 0 ntario français afin de contrer la violence conjugale. 


\section{Aperçu du programme d'intervention contre la violence conjugale}

Élément essentiel de la stratégie de criminalisation du M inistère des services sociaux et communautaires (1994), le programme d'intervention contre la violence conjugale, et plus particulièrement le programme pour hommes violents, est un moyen de faire face au problème de la violence contre lesfemmes. Ce moyen fait partie d'une stratégie globale visant à enrayer la violence conjugale. Ce programme est fidèle aux directives énoncées par le ministère des Services sociaux et communautaires (1994), et par le ministère des Services correctionnels, nos bailleurs de fonds, quant à la nature des services rendus et à leur qualité. Parmi ces directives, retenons les grands principes promulgués par le ministère des Services sociaux quant à ce programme:

1. La sécurité des femmes est primordiale.

2. L'agression est un crime. Toute personne, qu'il s'agisse du conjoint ou de quelqu'un d'autre, qui maltraite ou agresse une femme est passible d'une sanction juridique.

3. Les hommes abusifs sont responsables de leur comportement abusif. Par comportement abusif infligé aux femmes, il faut entendre l'abus physique, sexuel, psychologique et la menace de violence infligée par un homme à sa partenaire. Cela comprend toute forme de domination coercitive, notamment financière et juridique, la violence contre les biens pour lesquels la femme a des intérêts financiers ou affectifs et la violence faite aux enfants et aux animaux familiers. II s'agit également de violence infligée à domicile ou à l'extérieur, que les partenaires soient mariés ou non, qu'ils vivent ensemble ou soient séparés au moment de l'incident.

4. La violence faite aux femmes est un problème qui touche l'ensemble des communautés. La participation communautaire est donc essentielle à son élimination. 
5. L'équipe et le programme doivent refléter la composition de la communauté (race, culture, âge, aptitudes, pays d'origine et statut socio-économique).

6. Le programme doit être accessible. Par là, on entend que les services répondent aux besoins de tous les clients peu importe leurs âges, races, origines ethniques, religions, aptitudes physiques ou mentales et statuts socio-économiques et qu'ils vivent ou non dans les régions rurales ou éloignées.

Ce sont ces principes qui régissent les programmes offerts aux femmesviolentées et aux hommes violents, par le Service Familial. O n aura dès lors compris que pour le Service Familial, I'objectif du programme d'intervention contre la violence conjugale est d'éliminer la violence conjugale afin d'augmenter la sécurité des femmes et de promouvoir des relations saines. En ce sens, le programme poursuit les buts suivants.

1. O ffrir une intervention appropriée aux hommes violents et femmes violentées de préférence par l'intervention de groupe.

2. A méliorer l'accessibilité des services aux membres de la communauté locale en tenant compte de la diversité de la communauté dans la planification, la mise en oeuvre et l'évaluation du programme.

3. Favoriser la participation des femmes violentées ou de leurs représentantes dans tous les aspects du programme afin de gérer le développement et le déroulement du programme.

4. R especter en tout temps les standards, rendre des comptes aux agences et aux ministères et offrir un service accessible.

5. O ffrir de l'éducation et de l'information aux clients et à la communauté quant aux dynamiques de la violence conjugale et de leurs impacts.

C es quelques éléments mettent en relief le programme d'intervention contre la violence conjugale poursuivi par le Service Familial de Sudbury.Toutefois, ce service direct aux victimes ou à leurs agresseurs ne suffit pas à contrer la violence conjugale. En ce sens, le Service Familial s'implique dans la communauté afin de poursuivre cette mission. 


\section{Aperçu communautaire}

Le Service Familial, par son programme d'intervention contre la violence conjugale, a comme politique de s'impliquer dans la communauté afin de collaborer avec les services locaux de lutte contre la violence faite aux femmes, et les autres services, agences ou organismes communautaires: police, système judiciaire, services de probation ou de libération conditionnelle, afin d'offrir une réponse coordonnée et globale au problème de la violence conjugale.

Afin d'atteindre ce but, notre équipe privilégie les stratégies suivantes:

- tisser des liens avec la communauté;

- procéder à des consultations avec les services, les agences et les groupes sociaux et,

- développer, en collaboration, des initiatives afin de répondre au problème.

Pour tisser des liens étroits avec la communauté et l'ensemble des services pouvant intervenir auprès des victimes ou des agresseurs, le Service familial procède par consultations continuelles avec ses collaborateurs: représentantes ou représentants du ministère des Services correctionnels ainsi que des maisons d'hébergement Genevra H ouse (Sudbury) et $\mathrm{H}$ aven $\mathrm{H}$ ouse (région $\mathrm{M}$ anitoulin et Espanola), afin d'effectuer une coordination adéquate, d'offrir les services nécessaires à la clientèle commune, et d'évaluer les exigences en matière de responsabilité et d'accessibilité.

$Q$ uant à la consultation avec les différents services, elle s'exerce de façon trimestrielle avec les représentantes de $\mathrm{H}$ aven $\mathrm{H}$ ouse, G enevra H ouse, et du ministère des Services sociaux et communautaires. Ces rencontres sont coordonnées par la superviseure de programme au ministère des Services sociaux et communautaires.

Finalement, le Service Familial, par son programme d'intervention contre la violence conjugale, est impliqué dans les initiatives 
locales. Par exemple, le Service Familial est impliqué dans la Coalition de Sudbury contre la violence faite aux femmes qui vise à éliminer les conditions sociales, politiques, et économiques qui perpétuent la violence contre les femmes dans notre région. La C oalition a aussi comme mandat de s'assurer que les services locaux soient accessibles tout en supportant les ressources existantes, en identifiant les lacunes, et en promouvant la prévention par l'éducation et la conscientisation. N ous avons aussi à notre disponibilité certains outils communautaires (Sudbury Coordinating Committee A gainst W ife Assault (1992), Sudbury Sexual Assault A dvisory C ommittee (1991).

\section{Réalisations du programme}

Le programme d'intervention auprès des femmes violentées et des hommes agresseurs est passé de 171 demandes de service en 1991-1992, à 382 demandes en 1994-1995. Si, dans le passé, une longue période d'attente s'avérait obligatoire, celle-ci a été réduite en 1993, suite à une restructuration du programme. Présentement, les clients sont rejoints à l'intérieur d'une semaine suite à l'accueil de leur demande, et l'évaluation préalable à l'intervention faite en moins d'un mois.

Le groupe demeure le mode d'intervention privilégié par le Service Familial pour le programme contre la violence conjugale. D 'ailleurs, elle est fortement recommandée aux femmes (l'intervention individuelle pouvant être offerte lorsqu'elle est la plus appropriée), et obligatoire pour les hommes. Dans la dernière année, 6 groupes d'hommes (dont 3 sur l'île $M$ anitoulin), et 4 groupes de femmes ont bénéficié des programmes d'intervention.

De plus, l'équipe du programme d'intervention contre la violence conjugale du Service familial organise des forums de dis cussion, siège sur différents comités et organismes, prépare des outils de travail, etc. 


\section{Difficultés à surmonter}

Les difficultés rencontrées par l'équipe du Service Familial sont nombreuses. À l'heure actuelle, la plus importante est sans contredit le manque de ressources humaines. Le programme est victime des coupures budgétaires par le gouvernement. Si les conséquences sont nombreuses, l'impact le plus significatif touche directement la prestation de service. En effet, l'annonce récente du gouvernement $\mathrm{H}$ arris représente, pour le Service Familial, la perte d'un poste contractuel. Ceci signifie qu'il ne reste que 2,5 postes permanents pour desservir une population de plus de 160000 personnes. II devient alors très difficile de répondre aux principes énoncés par le ministère des Services sociaux et communautaires face à l'accessibilité des services à la clientèle. Le Service Familial offrant des services bilingues, il a tendance à offrir les interventions de groupes dans la langue la plus demandée. M alheureusement, l'anglais étant la langue de la majorité, il n'est pas toujours possible d'offrir nos services en français. Toutefois, l'agence a pris la décision d'offrir, pendant la saison hivernale, des groupes (pour hommes et femmes) en français. Par ailleurs, la population multiethnique est mal desservie. Lors des consultations avec le $M$ inistère, cette question revient toujours à l'ordre du jour. Ainsi, les compressions budgétaires imposantes entraînent dans leur sillon la question de la qualité versuscelle de la quantité des services. 0 r, le ministère préfère la seconde avenue. De plus, la possibilité que les programmes pour hommes violents soient éliminés, est à l'ordre du jour. Finalement, le Service Familial, en raison de son nombre restreint d'intervenantes et d'intervenants ne suffira pas à répondre à la demande, tout particulièrement dans les programmes offerts aux enfants, de prévention et d'éducation communautaire.

La seconde difficulté tient à ce que le Service Familial n'obtient pas toujours le soutien nécessai re à son programme d'intervention contre la violence conjugale. Tout particulièrement, il existe un manque de collaboration de la part du système judiciaire. 
C elui-ci a encore tendance à identifier la violence à un problème de gestion de colère, alors que, pour le Service Familial, il s'agit d'une dynamique de pouvoir et de contrôle exercée par le conjoint contre sa partenaire. Ainsi, même si le programme d'intervention pour les hommes violents est subventionné, en partie, par le ministère des Services correctionnels, et placé à sa disponibilité, les points de vue divergent entre ces deux organismes. Par exemple, malgré que toutes les récidives rapportées de violence de l'agresseur soient divulgués aux officiers de probation, aucune accusation additionnelle n'est portée contre ceux qui continuent à commettre des actes criminels. D e ce fait, le message transmis aux hommes et aux femmes est très clair, la violence demeure acceptable, car aucune mesure de représailles n'est prise.

II en va de même pour les sentences. À Sudbury, la peine rattachée à un acte de violence est habituellement de 18 mois de probation ou de 150 \$ d'amende. R écemment, un de nos clients a reçu une amende de 10 \$ pour avoir abusé physiquement sa conjointe. Finalement, l'agresseur n'est pas toujours référé au programme offert, ou encore il n'est référé qu'après plusieurs mois. $M$ algré la bonne relation que le Service Familial entretient avec les officiers de probation, ceux-ci nous disent qu'ils ne reçoivent pas l'appui des avocats ou des juges. Cette situation paradoxale devient un non sens, car l'objectif du programme est de promouvoir la sécurité des femmes.

La troisième difficulté tient à l'efficacité du programme d'intervention conjugale qui varie selon qu'il soit offert aux femmes ou aux hommes. D ans le programme offert aux femmes, on remarque que l'intervention offerte apporte des résultats concrets, observables et importants. Le programme atteint généralement ses objectifs d'encourager les femmes, d'assurer leur sécurité, de réduire leur sentiment de culpabilité, d'améliorer leur sentiment d'estime de soi, d'explorer et de développer des habilités afin de contrer la violence qu'elles subissent, etc. Des intervenantes telles K ilgore (1992), Larouche (1989, 1993), N iC arthy $(1984,1986)$ ou Sinclair (1985) ont construit des modèles solides d'intervention et de compréhension de la dynamique de la violence conjugale qui aident amplement dans le 
travail individuel ou de groupe. Par contre, du côté des hommes, les résultats de l'intervention sont moins positifs et, par conséquent, plus alarmants. Les constats sont multiples.

Du côté pratique, l'intervention auprès des hommes est un domaine relativement jeune. $E n O$ ntario, la référence théorique couramment utilisée est le modèle «D uluth» fondé par Ellen Pence (1990, 1993; Domestic Abuse Intervention Project, 1985; Pennsylvania C oalition Against Domestic Violence, 1985). Ce modèle américain est fondé sur la nécessité d'une relation étroite avec le système judiciaire et sur le principe de confrontation. $0 \mathrm{r}$, nous avons mentionné que la collaboration avec le système judiciaire est mince. $M$ algré les nombreuses tentatives faites par le Service Familial pour rencontrer les juges de la C our de Sudbury, ceux-ci n'ont jamais accepté l'invitation. De plus, il semble que les juges ne soient pas adéquatement informés et formés à la dynamique de la violence conjugale. Par conséquent, le modèle utilisé n'est pas aussi efficace car notre relation avec le système judiciaire n'est pas ce qu'elle devrait être. De plus, il semble évident que la confrontation n'est pas nécessairement un stimulant qui suscite le changement. D es études américaines sur le modèle «uluth» démontrent que malgré un fondement solide, il y a encore un taux élevé de récidives chez les hommes violents.

Afin de contourner la difficulté d'utiliser le modèle Duluth, des intervenants canadiens ont tenté d'implanter des modèles alternatifs (Pressman, 1989; R eberg, 1988; R ussel, 1994; Sonkin, 1985). Toutefois, le modèle d'intervention utilisé auprès des agresseursn'est pas uniforme à la grandeur de l'O ntario entrainnant une inconsistance dans le traitement offert à la population masculine. Plus précisément, en 0 ntario français, nous ne possédons aucun modèle théorique traitant de l'intervention auprès des hommes violents. Les modèles disponibles proviennent du Q uébec, et ils sont souvent forts différents de notre pratique.

A près plusieursannées d'intervention auprès de cette clientèle, le Service Familial de Sudbury a opté pour une approche qui oblige les hommes violentsà reconnaître la responsabilité de leurs actes (Jenkins, 1990). Les intervenantes et intervenants favorisent l'approche féministe, tout en appliquant certains concepts des 
modèles de la modification du comportement, de l'apprentissage social et de l'expression de soi, tout particulièrement lorsqu'il s'agit de discuter de leurs comportements et attitudes.

\section{Conclusion}

Les intervenantes et intervenants du Service Familial considèrent la violence conjugale comme un problème social, sur lequel on doit travailler de façon globale. En ce sens, notre expérience nous permet de constater que même si la violence conjugale est un problème social grave, la violence contre les femmes continue à être tolérée dans la communauté. $0 \mathrm{n}$ peut facilement prédire que l'intervention auprès des hommes violentsn'aura pas d'impact significatif, tant et aussi longtemps que la problématique de la violence conjugale ne sera pas soutenue et favorisée à toutes les instances sociales. U ne intervention plus large, incluant les programmes pour femmes violentées, hommes agresseurs, prévention et éducation, est primordiale afin d'éliminer la violence conjugale.

Ce que les intervenantes et intervenants du Service Familial vivent dans le programme d'intervention pour contrer la violence conjugale n'est pas exclusif à Sudbury. En discutant avec des collègues de différents services de la province, presque toutes et tous disent vivre les mêmes réalités et frustrations.

Ainsi, l'intervention auprès des femmes est bénéfique, même si la majorité de nos clientes continuent d'être violentées lorsqu'elles restent avec leur conjoint violent. En effet, nous pouvons pointer les changements concrets dans leurs attitudes, leur compréhension de la dynamique de la violence ou dansleur estime de soi. Par contre, quant à l'intervention auprès des agresseurs, aucune recherche ne démontre, jusqu'à maintenant, des résultats concrets qui soient positifs à long terme. II y a un manque flagrant de financement à la recherche et à l'expérimentation de nouveaux modèles, et d'efforts de promotion de 
la non-violence par les médias. La perpétuation de la violence, la diffusion médiatisée de la violence sous toutes ses formes, et l'accroissement du nombre de cas de violence conjugale ne représentent pas un problème clinique ou d'intervention mais une réalité sociale inquiétante.

Conséquemment, l'intervention sociale et la collaboration communautaire demeurent les outils essentiels si nous visons à l'élimination de la violence conjugale. Les pratiques du gouvernement $\mathrm{H}$ arris démontrent une absence d'intérêt envers la problématique de la violence conjugale. Les récentes compressions budgétaires, de même celles qui s'annoncent à l'avenir, envoient un message clair et troublant tant leurs conséquences sont graves pour venir à bout de la violence conjugale.

\section{Bibliographie}

DIR ECTION GÉNÉR ALE DE LA CON DITION FÉM IN IN E DE L'ON TAR IO (1993). Forum: Pour la séaurité des femmes, Toronto, $D$ irection générale de la conditions féminine de l'O ntario.

DOM EST IC A BUSE IN TERVEN TIO N PRO JEC T (1985). T he justice system's response to domestic assault cases: A guide for policy development, (document ronéotypé).

HART, Barbara (1988). Safety for women: M onitoring batterers' programs, Harrisburg, Pennsylvania Coalition Against D omesticV iolence.

HART, Barbara (1992). A countability: P rogram standards for batterer intervention services, R eading, Pennsylvania, C oalition Against D omestic Violence.

JEN K IN S, A Ian (1990). Invitations to responsibility:T he therapeutic engagement of men who are violent and abusive, Adelaide, Dulwich Centre Publications.

KILGOR E, N ancy (1992). Sourcebook for working with battered women, Volcano,Volcano Press.

LAR O U CHE, Ginette (1989). A gir contre la violence, M ontréal, La pleine Lune.

LAR O U CHE, Ginette (1993). A ux formatrices en intervention auprès des femmes violentées, M ontréal, $W$ ilson et Lafleur Ltée.

M IN IST ÈR E DES SERVICES SO CIAU X ET CO M M U N AU TAIR ES (1994). Exigences provisoires en matière de responsabilité et d'accessibilité des programmes d'aide aux hommes agresseurs, Toronto, $M$ inistère des services sociaux et communautai res.

N IC ARTHY, Ginny, Karen MER R IAM et Sandra COFFM AN (1984). Talking it out: A guide for groups for abused women, Washington, Seal Press.

N IC ARTHY, Ginny (1986). G etting free:You can end abuse and take back your life, Washington: Seal Press.

PEN CE, Ellen et M ichael PAYMAR (1990). Power \& control: Tadics of men who batter, (document ronéotypé). 
PEN CE, Ellen et M ichael PAY M AR (1993). E ducation groups for men who batter:T he D uluth model, N ew York, Springer Publishing C ompany.

PEN NSY LVAN IA COALITION AGAINST DOMESTIC VIO LENCE (1985). C hanging police practice: A manual for battered women's programs, (document ronéotypé)

PR ESSM AN, Barbara, Gary CAM ERON et M ichael ROTHERY (dir.) (1989). Intervening with assaulted women: $C$ urrent theory, research, and practice, Law rence Erlbaum Associates.

R EBER G, Douglas (1988). The G roup D elta H andbook: A manual to help men stop family violence, (document ronéotypé).

RU SSEL, M ary N omme et Jobst FR O H BER G (1994). C onfronting abusive beliefs: A group treatment program for men who abuse their partners,Vancouver.

SI N C LAIR , D eborah (1985). P our mieux comprendre le problème des femmes battues: $G$ uide de formation pour les conseillers et les intervenants, Toronto, Education W ife A ssault.

SO N K IN , D aniel Jay et M ichael D U R PHY (1985). L earning to live without violence,Volcano,Volcano Press.

SU DBURY COOR DINATIN G COM MITTEE AGAIN ST W IFE ASSAU LT (1992). Linking our steps: A resource manual for service providers working with assaulted women, their children and men, Sudbury, Sudbury C oordinating C ommittee A gainst W ife A ssault.

SU DBURY SEX UAL ASSAULT ADVISORY COM M ITTEE (1991). A community response/ U ne initiative communautaire, Sudbury, Sudbury Sexual A ssault A dvisory Committee. 